\title{
Timely completion of vaccination and its determinants among children in northwest, Ethiopia: a multilevel analysis
}

\author{
Zeleke Abebaw Mekonnen ${ }^{1,2^{*}}$ D, Kassahun Alemu Gelaye ${ }^{3}$, Martin C. Were ${ }^{4}$ and Binyam Tilahun ${ }^{1}$
}

\begin{abstract}
Background: Timely vaccination is key to prevent unnecessary childhood mortality from vaccine-preventable diseases. Despite the substantial efforts to improve vaccination completeness, the effort towards timeliness of vaccination is limited with non-attendance and delays to vaccination appointments remaining a big challenge in developing countries. There is also a limited evidence on timeliness of vaccination. Therefore, this study aimed to determine the magnitude and associated factors for timely completion of vaccination among children in Gondar city administration, north-west, Ethiopia.
\end{abstract}

Method: This study employed a community-based cross sectional study design. A sample size of 821 children aged 12 to 23 months were considered. Two stages random sampling technique was used to select study subjects. To account the effect of clustering, bivariable and multivariable multilevel logistic regression analysis were applied. The measures of association estimates were expressed as adjusted odds ratio (AOR) with their $95 \%$ confidence intervals (Cls).

Results: Of the 774 children included for analysis, 498 (64.3\%) were fully vaccinated while 247 (31.9\%) were fully vaccinated on-time. Caregivers who had secondary education and above ( $\mathrm{OOR}=2.391$; 95\% Cl: 1.317-4.343), from richest households ( $\mathrm{AOR}=2.381 ; 95 \% \mathrm{Cl}$ : 1.502-3.773), children whose mother attended four or more ante natal care visits (AOR $=2.844 ; 95 \% \mathrm{Cl}: 1.310-6.174)$ and whose mother had two or more post natal care visits (AOR= 2.054; 95\%Cl:1.377-3.063) were positively associated with on-time full vaccination. In contrary, caregivers aged above 35 years ( $A O R=0.469 ; 95 \% \mathrm{Cl}: 0.253-0.869$ ], being vaccinated at health post (AOR $=0.144 ; 95 \% \mathrm{Cl}: 0.048-$ 0.428 ) and travelling more than $30 \mathrm{~min}$ to the vaccination site ( $\mathrm{AOR}=0.158 ; 95 \% \mathrm{Cl}$ : $0.033-0.739$ ) were negatively associated with on-time full vaccination. The random effects indicated that $26 \%$ of the variability in on-time full vaccination was attributable to differences between communities.

Conclusion: In this study, untimely vaccination was found to be high. Different individual and contextual factors were found to be associated with on-time full vaccination. Therefore, tailored strategies have to be designed and implemented to address people and the communities where they live. Moreover, timeliness of vaccination should be considered as important indicator of the immunization program performance in Ethiopia.

Keywords: Vaccination, Immunization, Timeliness, Completeness, Ethiopia

\footnotetext{
* Correspondence: Zelekeabebaw7@gmail.com

'Department of Health Informatics, Institute of Public Health, University of Gondar, Gondar, Ethiopia

${ }^{2}$ Health System Directorate, Federal Ministry of Health, Addis Ababa, Ethiopia

Full list of author information is available at the end of the article
}

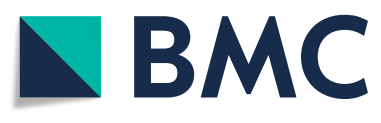

(c) The Author(s). 2020 Open Access This article is licensed under a Creative Commons Attribution 4.0 International License, which permits use, sharing, adaptation, distribution and reproduction in any medium or format, as long as you give appropriate credit to the original author(s) and the source, provide a link to the Creative Commons licence, and indicate if changes were made. The images or other third party material in this article are included in the article's Creative Commons licence, unless indicated otherwise in a credit line to the material. If material is not included in the article's Creative Commons licence and your intended use is not permitted by statutory regulation or exceeds the permitted use, you will need to obtain permission directly from the copyright holder. To view a copy of this licence, visit http://creativecommons.org/licenses/by/4.0/. The Creative Commons Public Domain Dedication waiver (http://creativecommons.org/publicdomain/zero/1.0/) applies to the data made available in this article, unless otherwise stated in a credit line to the data. 


\section{Background}

Vaccine preventable diseases contribute substantially to under five mortality as well as morbidity [1]. Hence, immunization has become one of the major contributors to public health globally as it prevents communicable disease, particularly in children. Evidence shows that $29 \%$ of deaths among under five children are vaccine preventable [2] and being fully vaccinated is associated with $22 \%$ lower mortality in children [3].

Immunization averts an estimated 2 to 3 million deaths every year worldwide. However, despite the tremendous efforts made so far, vaccine preventable diseases remain a major health problem among children in developing countries including Ethiopia [4-7].

Completion and timeliness of routine vaccination as recommended by the World Health Organization (WHO) are very crucial for maximum protection of children against specific infections [8]. To effectively control vaccine preventable diseases (VPDs), high immunization coverage is required with a targeted coverage level of $90 \%$ recommended by the WHO. In terms of control and prevention of childhood infectious diseases, achieving high vaccination coverage is a necessary; but insufficient indicator to assess the success of immunization program. In order to successfully control and eliminate vaccine-preventable infectious diseases, age appropriate vaccine coverage has to be achieved and maintained [912].

However, maintaining a high performance of an immunization program and its quality is challenging, with non-attendance and delays to vaccination appointments remaining a key challenge in developing countries [13, 14]. Worldwide in 2018, about 116.3 million infants (86\%) received three doses of diphtheria-tetanuspertussis (DTP3) vaccine, protecting them against infectious diseases. On the other hand, an estimated 19.4 million infants worldwide were not reached with routine immunization services of which around $60 \%$ of these children live in 10 countries, including Ethiopia [15]. As a result, substantial proportions of children in many countries still fail to benefit from all basic vaccines and vaccine preventable diseases still pose a public health risk with the highest rates of child mortality still in SubSaharan Africa [16, 17].

Ethiopia launched the immunization program in 1980 and currently provides 11 antigens at birth, 6 weeks, 10 weeks, 14 weeks and 9 months. Despite the fact that immunization services are offered free of charge, full vaccination coverage in Ethiopia is significantly lower than the global target. The 2016 Ethiopian Demographic and Health Survey (EDHS) report showed that only 39\% of children received all basic vaccinations [18] and the 2019 mini EDHS report also indicated that the full vaccination coverage has reached $43 \%$ with steady increase in vaccination coverage over time [19]. Findings from studies conducted in different settings of Ethiopia showed a wide range coverage of full vaccination from 48.8 to $91.7 \%$ [20-26]. On the other hand, the incomplete vaccination coverage ranged from 20.3 to $45.5 \%$ $[20,23,24]$.

Multiple assessments also reveal that despite relatively high vaccination coverage, there was a gap in the timeliness of children vaccination with substantial delay in age-appropriate vaccination demonstrating that high vaccination coverage does not imply that children are vaccinated according to the schedule [16, 27-36].

Currently, the timing of vaccine administration has received increasing attention in many countries, especially when the level of vaccination coverage is close to that needed for protective herd immunity [10, 29, 37]. Although many studies have measured full vaccination coverage, studies on timely vaccination completion are rare in developing countries particularly in Sub- Saharan Africa (SSA) [16, 31].

To date, timeliness is not routinely used as an indicator to evaluate immunization programs in Ethiopia. Similarly, several studies had examined and documented the vaccination coverage for childhood immunization in Ethiopia with which timeliness had received less consideration [13, 19, 22, 24, 38]. Therefore, this study aimed to determine the coverage and associated factors for timely completion of vaccination among children in Gondar city administration, Northwest, Ethiopia.

\section{Methods}

\section{Study design, area and period}

A community based cross sectional study was conducted from October 22 to November 30, 2018 in Gondar city administration, northwest, Ethiopia. The city administration had an estimated total population of 390, 644 of which 12,149 were under 1 year of age. Gondar city administration has a total of 24 Kebeles (13 urban and 11 rural). In addition, the city administration has a total of 23 public health facilities (one comprehensive specialized hospital, eight health centers and 14 health posts [39].

\section{Source and study population}

The source population were all children aged 12 to 23 months with a history of routine vaccination in Gondar city administration. The study population were those children aged 12 to 23 months with their caregivers living in the eligible households of the selected kebeles and included for this particular study.

\section{Inclusion and exclusion criteria}

Households with at least one live child aged 12 to 23 months and who resided in the study area for at least 6 months prior to the study period were eligible for 
inclusion in this study. Those children included in this study had a history of routine vaccination. Children having history of vaccination from vaccination campaigns only were excluded.

\section{Sample size determination}

The required sample size was calculated for both completeness and timeliness of vaccination using single population proportion formula by considering the following assumptions:

- Using proportion of $58.7 \%$ for full vaccination [Pilot study], $95 \%$ confidence level, $5 \%$ margin of error, design effect of 2 and non-response rate of $10 \%$, the sample was 821.

- Using proportion of $37.1 \%$ for on time-full vaccination [Pilot study], 95\% confidence level, 5\% margin of error, design effect of 2 and non-response rate of $10 \%$, the sample was 395 .

Hence, we used a sample size of 821 for this particular study.

\section{Sampling procedure}

Two stages sampling technique was used. During the first stage, from the 24 kebeles (13 urban and 11 rural) $40 \%$ of the kebeles were considered to be included in this study. From the total kebeles in the city administration, five urban and five rural kebeles were selected proportionally from each stratum using simple random sampling technique.

In the second stage of sampling, at each selected kebele, individual households were selected using systematic random sampling technique. Children in the selected households were further selected. If there were two or more children in the same household, lottery method was used to select one child per household. When there was no eligible child in the selected household, the next household was considered in the study.

\section{Study variables measurement \\ Dependent variable}

On-time full vaccination.

\section{Independent variables}

Socio-demographic characteristics of the caregiver, health service related characteristics of the mother and contextual factors at community level were considered as independent variables for this study.

Full vaccination was defined as the child vaccination status once an infant has received all recommended vaccines included in the national schedule: a dose of Bacille Calmette Guérin (BCG); three doses of Oral Polio Vaccine (OPV); three doses each of Penta-valent and
Pneumococcal Conjugate Vaccine (PCV); one dose of Inactivated Polio Vaccine (IPV); two doses of rotavirus and one dose of measles vaccines by the age of 12 months $[5,22,26,40,41]$. On-time vaccination for specific vaccines was defined as vaccine dose administered within 4 days prior $[31,42-45]$ and within 4 weeks after the recommended age specified in the national immunization schedule [12, 30, 31, 37, 43-49]. On-time full vaccination was also defined as all vaccine doses administered within 4 days prior [31, 42-45] and within 4 weeks after the recommended age specified in the national immunization schedule. Otherwise, it was not considered as on-time full vaccination if at least one vaccine dose was given early, late or missed at all [30, 31, $43,44,48,50-53]$.

The household socio-economic status was created by principal components analysis (PCA), including variables on asset ownership, housing characteristics and ownership of animals and farming. This was done for rural and urban households separately [54]. Having this, rural and urban households PCA loading scores were merged for household wealth index classification using quintiles. Finally, the merged scores for urban and rural were divided into three quintiles as poor, middle and rich households.

\section{Data collection tools and procedures}

Data collection instrument was adapted from EDHS [18]. Face and content validity has been ensured by a group of six experts. Accordingly, the applicability of the data collection tools and procedures were checked and revised as necessary.

Pilot study was also done out of the study area (in four kebele's of Bahirdar city administration) before the actual data collection with a sample size of 100 . The results of the pilot study were used to determine the minimum sample size for the actual study. The reliability of the data collection instrument was assessed using Cronbach's alpha $(\alpha)$. From the pilot study the internal consistency estimate for the full vaccination scale was found to be 0.87 .

Interviewer-administered data collection instrument was used to collect socio-demographic characteristics, health service related factors and vaccination status of the children. Eight data collectors and two supervisors were recruited for the data collection. Vaccination status and age at vaccination were confirmed by checking the vaccination card kept by caregivers or from the health facility expanded program on immunization (EPI) registers. For children with a vaccination card, the interviewer copied dates of any recorded vaccination on to the data collection instrument. For children without a vaccination card, their vaccination status was verified from the health facility EPI registers. 
Data processing, analysis and parameter estimation methods

\section{Descriptive statistics}

The data were entered into EPI-data version 3.1 software and transferred to STATA version 14 software for analysis. Prior to the commencement of the analysis data cleaning, labeling, coding and recoding were done for all variables. Frequency and percentages were used to report categorical variables.

Bivariable and multivariable multilevel regression analysis At the bivariable multilevel regression analysis, the effect of each individual and community level predictor variables on the outcome variable were checked at significance level of 0.2 [55]. Variables which were significant at the bivariable multilevel logistic regression analysis were considered as candidates for the individual and community level model adjustments. Finally, a significance level of 0.05 was considered for the multivariable multilevel regression models.

\section{Model specification}

This study applied binary logistic multilevel analysis techniques in order to account for the clustering nature of the data and the binary response of the outcome variable. For the bivariable and multivariable multilevel logistic regression analysis the STATA syntax xtmelogit was used. Accordingly, four models containing variables of interest were fitted.

Model-I: was the null model, used to check the variability among the communities without inserting any variable. It's the first step used to provide evidence whether the data has a justifiable evidence to assess the random effects at the community level. Model-II: was a multivariable model used to adjust individual level variables which were significant at the bivariable multilevel regression analysis. Hence, independent variables which were significant in Model-II were considered as candidates of the final model. Model-III: was also a multivariable model which was used to adjust community level variables which were significant at the bivariable multilevel regression analysis. Community level independent variables which were statistically significant in Model-III were included in the final model. Model-IV: was a multivariable multilevel regression analysis model used to adjust the outcome variable against independent variables which were statistically significant either at Model-II or Model-III. Stepwise model building technique was used for all models.

\section{Parameter estimation methods}

The measures of association (fixed-effects) estimates the association between the likelihood of children to be fully vaccinated on-time and the predictor variables expressed as Adjusted Odds Ratio (AOR) with their 95\% Confidence Intervals (CIs). The measures of variation (random-effects) were reported as intra class correlation coefficient (ICC) which is the percentage of variance explained by the community level variables. Proportional Change in Variance (PCV), expresses the change in the community level variance between Model-I (empty model) and the consecutive models (Model-II, III and IV) [56].

\section{Multicollinearity and interaction effect}

The presence of multicollinearity was checked among independent variables using Variance Inflation Factor (VIF) at cut off point of 10 [57]. Similarly, interaction terms between community and individual level variables were tested.

\section{Comparison of models and model fit statistics}

Akakie Information Criterion (AIC) was used to compare the consecutive models. The AIC values for each subsequent models were compared and the model with the lowest value was considered to be the better model [58]. Finally, Hosmer-Lemeshow goodness of fit test was used to estimate the goodness of fit of the adjusted final model [55].

\section{Ethical considerations}

Ethical approval was obtained from the University of Gondar Ethical Review Board (IRB) before the commencement of the study. In addition, study permission was sought at all levels of local governmental health administrations. Informed written consent was obtained from each of the caregivers of children for their participation in the study and to access their child vaccination data from health facilities. In the meantime, study participants were informed to withdraw and discontinue participation at any time if they felt discomfort. Moreover, confidentiality assurance was provided to study participants on the information provided by them. Information that was collected for this study was also secured and protected from unauthorized access. At last, official permission was requested from the health facilities to access the child vaccination records from EPI registers.

\section{Results}

\section{Socio-demographic characteristics of caregivers}

Out of the 821 children included for this study, the response rate was $98.9 \%$. Thirty eight children with unverifiable records were excluded and a total of 774 children were included for analysis. Almost all (97.8\%) of the respondents as a primary caregiver were mothers of the eligible child and $475(61.4 \%)$ were in the age range of 25-34 years. About, 67\% of respondents were from urban kebeles. Pertaining to educational status, majority 
(46\%) had secondary education and above while $22 \%$ had no education. As indicated in Table 1, 693 (89.5\%) of the caregivers were married and 500 (64.6\%) were housewife by occupation. With regard to sex of child, $406(52.4 \%)$ of children were males [Table 1].

\section{Health service related characteristics of mothers/ caregivers}

Majority 469 (60.6\%) of caregivers reported that the mother had four or more ante natal care (ANC) visits for the child included for this study. Regarding place of

Table 1 Socio-demographic characteristics of caregivers in Gondar city administration, north-west Ethiopia, 2018 [N = 774]

\begin{tabular}{lr}
\hline Characteristics & Total (\%) \\
\hline
\end{tabular}

\section{Caregiver age in years}

$\leq 24$

25-34

$\geq 35$

\section{Marital status}

Married

Others

\section{Religion}

Orthodox

Muslim

Others

Residence

Rural

Urban

\section{Education}

No formal education

Primary

Secondary and above

\section{Sex of child}

Female

Male

\section{Occupation}

Housewife

Employed

Merchant

Others

Family size

$<5$

$\geq 5$

Wealth Index

Poor

Middle

Rich
$172(22.2)$

475 (61. 4)

$127(16.4)$

693(89.5)

$81(10.5)$

707(91.4)

$53(6.8)$

$14(1.8)$

252(32.6)

522(67.4)

171(22.1)

244(31.5)

359(46.4)

368(47.6)

406 (52.4)

$500(64.6)$

88 (11.4)

115(14.9)

$71(9.1)$

436 (56.3)

338(43.7)

$258(33.3)$

258 (33.3)

$258(33.3)$ delivery, only 54(6.9\%) of deliveries were at home. Sixty eight percent of respondents reported that the mother of the child had two or more post natal care (PNC) visits. Of the included children for the study, 279 (36.1\%) were in the first birth order. Four hundred and ninety-two (63.6\%) of the caregivers took their child to a health center for vaccination and 391 (50.5\%) of respondents reported that the distance to the vaccination site was less than 15 min from their home (Table 2).

\section{Over all vaccination status of children}

Child vaccination card availability during the time of interview was 599 (77.4\%) (Fig. 1). Of those caregivers who reported that their child's vaccination card is available during the time of interview, 551 (91.9\%) showed the vaccination cards for the interviewers.

Of the 774 children included for analysis, 498 (64.3\%) with 95\%CI: (60.9-67.6\%) were fully vaccinated while 247 (31.9\%) with 95\% CI (28.7-35.3\%) children were fully vaccinated on-time (Fig. 1).

Table 2 Health service related characteristics of mothers/ caregivers in Gondar city administration, north-west Ethiopia, $2018[N=774]$

\begin{tabular}{ll}
\hline Variables & Total (\%) \\
\hline ANC & $61(7.9)$ \\
No & $244(31.5)$ \\
$1-3$ & $469(60.6)$ \\
Place of delivery & \\
Home & $54(6.9)$ \\
Health Facility & $720(93.1)$ \\
PNC & \\
$<2$ & $243(31.4)$ \\
$\geq 2$ & $531(68.6)$ \\
Birth order & \\
1st & $279(36.1)$ \\
2nd-3rd & $347(44.8)$ \\
$4+$ & $148(19.1)$ \\
Distance to vaccination site & \\
$<15$ Minute & $391(50.5)$ \\
15-30 Minute & $309(39.9)$ \\
$>30$ Minute & $74(9.6)$ \\
Place of vaccination & $130(16.8)$ \\
Hospital & $152(19.6)$ \\
Health post & $492(63.6)$ \\
\hline
\end{tabular}




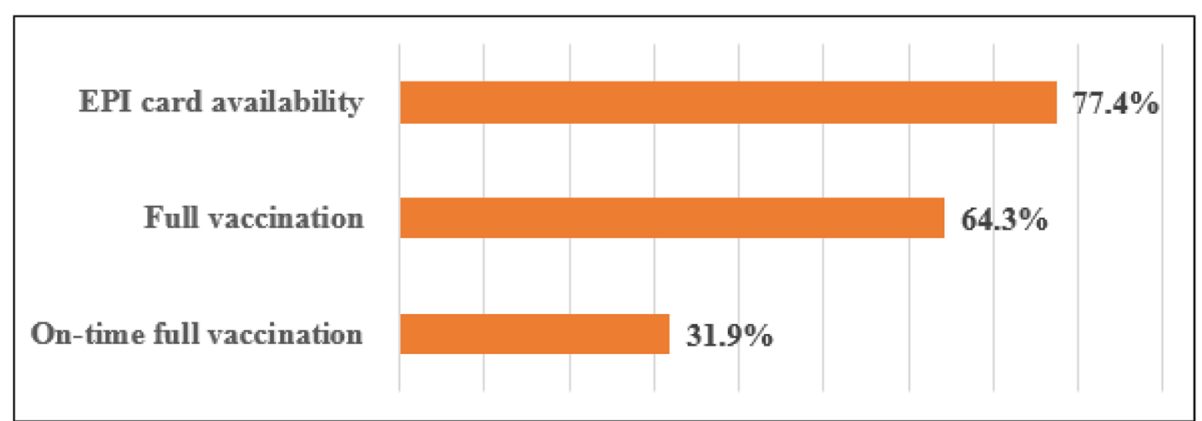

Fig. 1 EPI card availability and vaccination status of children in Gondar city, north-west Ethiopia, 2018 [N=774]

\section{Vaccination coverage for specific vaccines}

Figure 2 below depicted the vaccination coverage for specific vaccines. Coverage for each specific vaccine was calculated from all children included in this particular study. We found that the proportion of children with full vaccinations decreased from Penta I (95.5\%) to Penta III (83.2\%) and measles (76.2\%) vaccine doses subsequently (Fig. 2). The study also indicated that the Pentavalent vaccination drop-out rate was $12.8 \%$ and the BCG to measles vaccination dropout rate was $20.1 \%$.

\section{Timely vaccination for specific vaccines}

Timeliness for each specific vaccine was calculated from those children vaccinated for that specific vaccine. As depicted in Fig. 3, timely vaccinations for each vaccine ranged from $62.4 \%$ for BCG vaccine to $80.5 \%$ for Rota I vaccine. The proportion of children who had received early vaccine doses ranged from $3.1 \%$ for PCV3 vaccine to $13.6 \%$ for measles vaccine. On the other hand, the proportion of children who had received vaccine doses lately ranged from 13.9\% for Rota1 vaccine to $37.6 \%$ for $\mathrm{BCG}$ vaccines (Fig. $3)$.

\section{Attendance to vaccination schedules}

Full attendance to vaccination schedules were measured historically by asking the caregivers and objectively measured from vaccination cards and registers. The findings showed that the proportion of full attendance to vaccination schedules measured historically from caregiver's report was 693(89.5\%). On the other hand, the objective measurement from vaccination cards and registers indicated that proportion of full attendance to vaccination visits was $558(72.1 \%)$.

\section{Reasons for not attending vaccination schedules on-time}

The reasons for not attending vaccination schedules were mentioned by those 81 caregivers who reported that their attendance to the vaccination schedules were not complete as scheduled. Among the reasons for not attending vaccination schedules on-time, $34.5 \%$ were due to forgetfulness, $28.4 \%$ being unaware of the

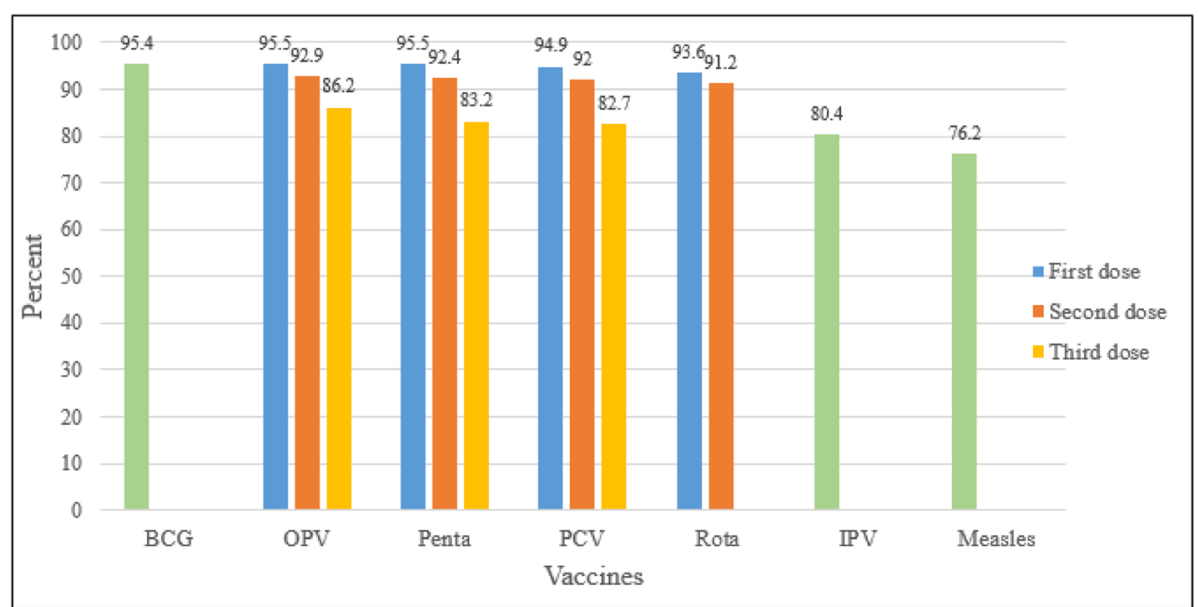

Fig. 2 Vaccination coverage for specific vaccines among children in Gondar city, north-west Ethiopia, 2018 [N=774] 


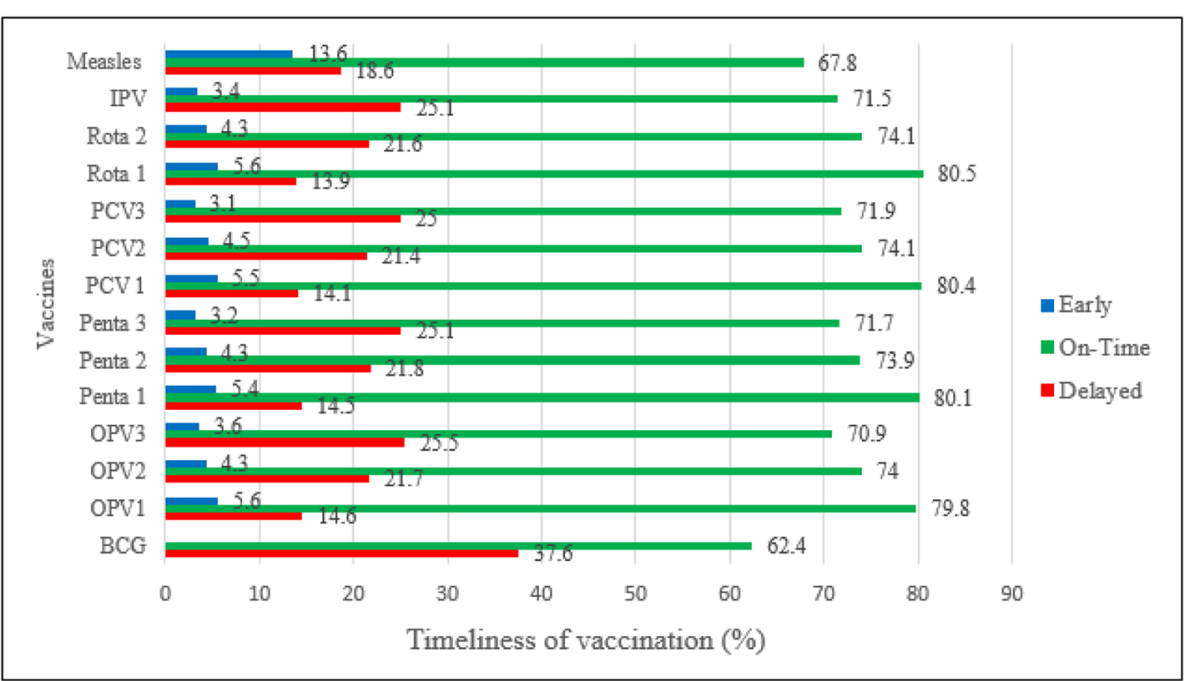

Fig. 3 Timeliness of vaccination for specific vaccines among children in Gondar city, north-west Ethiopia, 2018

schedules and $27 \%$ being busy with other engagements to show up in vaccination schedules (Fig. 4).

\section{Multilevel logistic regression analysis}

The fixed and random effects for on-time full vaccination are presented in Table 3. The ICC in the empty model implied that $25.7 \%$ of the total variance in ontime full vaccination was attributed to differences between communities (Table 3).

In Model-II only individual level variables were added. In this model the variables age of caregiver, marital status, religion, occupation, family size, sex of child, caregiver education, birth order, ANC, place of delivery, PNC and wealth index were included. With this, marital status, religion, family size and sex of child were insignificant at the bivariable regression analysis. Finally, the variables occupation, birth order and place of delivery were statistically insignificant at Model-II. The results showed that caregiver's age, caregiver's education level, household wealth index, antenatal care visits and post natal care visits were significantly associated with ontime full vaccination in Model-II. The ICC in Model-II indicated that, $23.5 \%$ of the variation in on-time full vaccination was attributable to differences across communities. As shown by the PCV, $11.4 \%$ of the variance in ontime vaccination across communities was explained by the individual level characteristics (Table 3).

In Model-III only community level variables were added. In model-III the community level characteristics residence, distance to vaccination site and place of vaccination were included. At the bivariable regression analysis all the three variables were statistically significant. In model-III, the variable place of residence became statistically insignificant. The results in Model-III revealed that place of vaccination and distance to the vaccination site were significantly associated with on-time full

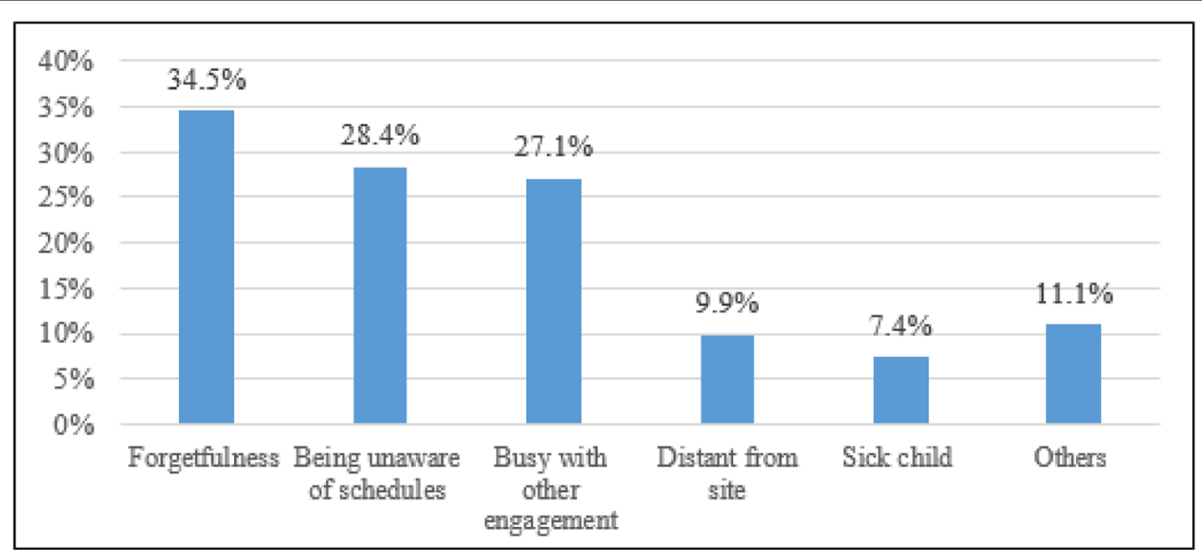

Fig. 4 Reasons for not attending vaccination schedules on-time in Gondar city, north-west Ethiopia, 2018 [N= 81] 
Table 3 Multilevel regression analysis of factors associated with on-time full vaccination in Gondar city, north-west Ethiopia

\begin{tabular}{|c|c|c|c|c|c|}
\hline \multicolumn{2}{|c|}{ Fixed effects of individual and community level variables } & \multirow[t]{2}{*}{ Model-I } & \multirow{2}{*}{$\frac{\text { Model-II AOR [95\%Cl] }}{1}$} & \multirow{2}{*}{$\begin{array}{l}\text { Model-III AOR [95\%Cl] } \\
-\end{array}$} & \multirow{2}{*}{$\frac{\text { Model-IV AOR }[95 \% C l}{1}$} \\
\hline Age of caregivers & $\leq 24$ years & & & & \\
\hline & $25-34$ years & & $1.012[0.669,1.531]$ & - & $1.031[0.681,1.561]$ \\
\hline & $\geq 35$ years & & $0.441[0.238,0.813]$ & - & $0.469[0.253,0.869]$ \\
\hline \multirow[t]{3}{*}{ Education of caregivers } & No education & & 1 & - & 1 \\
\hline & Primary & & $1.901[1.012,3.571]$ & - & $1.786[0.954,3.343]$ \\
\hline & Secondary and above & & $2.587[1.407,4.756]$ & - & $2.391[1.317,4.343]$ \\
\hline \multirow[t]{3}{*}{ Wealth Index } & Poor & & 1 & - & 1 \\
\hline & Middle & & $1.419[0.915,2.201]$ & - & $1.494[0.976,2.287]$ \\
\hline & Rich & & $2.269[1.419,3.627]$ & - & $2.381[1.502,3.773]$ \\
\hline \multirow[t]{3}{*}{ ANC } & No & & 1 & - & 1 \\
\hline & $1-3$ & & $1.416[0.627,3.201]$ & - & $1.404[0.618,3.191]$ \\
\hline & $4+$ & & $3.139[1.456,6.764]$ & - & $2.844[1.310,6.174]$ \\
\hline \multirow[t]{2}{*}{ PNC } & $<2$ & & 1 & - & 1 \\
\hline & $\geq 2$ & & $2.067[1.373,3.109]$ & - & $2.054[1.377,3.063]$ \\
\hline \multirow[t]{3}{*}{ Place of vaccination } & Hospital & & - & 1 & 1 \\
\hline & $\mathrm{HP}$ & & - & $0.103[0.036,0.289]$ & $0.144[0.048,0.428]$ \\
\hline & $\mathrm{HC}$ & & - & $0.809[0.511,1.282]$ & $1.011[0.612,1.671]$ \\
\hline \multirow[t]{3}{*}{ Distance to vaccination site (minute) } & $<15 \min$ & & - & 1 & 1 \\
\hline & $15-30 \mathrm{~min}$ & & - & $0.661[0.469,0.933]$ & $0.746[0.516,1.080]$ \\
\hline & $>30 \min$ & & - & $0.155[0.034,0.701]$ & $0.158[0.033,0.739]$ \\
\hline \multicolumn{6}{|l|}{ Random effects } \\
\hline Random effect & & Model-I & Model-II & Model-III & Model-IV \\
\hline Community variance (SE) & & $1.14(0.62)$ & $1.01(0.59)$ & $0.069(0.08)$ & $0.079(0.093)$ \\
\hline ICC (\%) & & $25.7 \%$ & $23.5 \%$ & $2.1 \%$ & $2.4 \%$ \\
\hline PCV (\%) & & Ref & $11.4 \%$ & $93.9 \%$ & $93.1 \%$ \\
\hline \multicolumn{6}{|l|}{ Model comparison statistics } \\
\hline Model comparison & & Model-I & Model-II & Model-III & Model-IV \\
\hline Log likelihood & & -447.77844 & -407.19174 & -432.57621 & -393.93011 \\
\hline AIC & & 899.5569 & 836.3835 & 877.1524 & 817.8602 \\
\hline
\end{tabular}

vaccination. The ICC in Model-III implied that differences between communities account for about $2.1 \%$ of the variation in on-time full vaccination. In addition, the PCV indicated that $93.9 \%$ of the variation in on-time full vaccination between communities was explained by community level characteristics (Table 3).

Model-IV, the final model included both the individual and community level characteristics simultaneously. After controlling for other individual and community level factors, caregivers aged above 35 years were $53 \%$ less likely $(\mathrm{AOR}=0.469 ; 95 \% \mathrm{CI}$ : $0.253-0.869$ ] to complete their child vaccination ontime as compared to those caregivers aged 25 years and less. The study also indicated that caregivers who had secondary education and above were 2.4 times $(\mathrm{AOR}=2.391 ; 95 \% \mathrm{CI}: 1.317-4.343)$ more likely to complete their child vaccination on-time as compared to those who had no education after controlling for other variables. After holding other factors constant, caregivers from richest households had 2.4 times higher chance of completing their child vaccination on-time (AOR $=2.381 ; 95 \% \mathrm{CI}: 1.502-3.773)$ as compared to caregivers from poorest households (Table $3)$.

Looking at ANC, children whose mothers had attended four and above ante natal care visits were 2.8 times $(\mathrm{AOR}=2.844 ; 95 \% \mathrm{CI}: 1.310-6.174)$ more likely to complete their child vaccination on-time as compared to those children whose mothers had no antenatal care checkups. Keeping other variables constant, children whose mothers had two and more PNC visits were 2 times more likely (AOR $=2.054 ; 95 \% \mathrm{CI}: 1.377-3.063$ ) to fully vaccinate their child on-time as compared to their counterparts (Table 3 ). 
Pertaining place of vaccination, those caregivers who vaccinated their child at health posts were $86 \%$ (AOR = 0.144; $95 \% \mathrm{CI}: 0.048-0.428$ ) less likely to fully vaccinate their child on-time as compared to those who vaccinated their child in hospital. In terms of distance to vaccination site, those caregivers who travelled more than 30 $\min$ to the vaccination site were $84 \%$ ( $A O R=0.158$; 95\%CI: $0.033-0.739)$ less likely to fully vaccinate their child on-time as compared to those who travelled less than 15 min to the vaccination site (Table 3).

As shown by the estimated ICC in model-IV, 2.4\% of the variability in on-time full vaccination was attributable to differences between communities. The PCV indicated that, $93.1 \%$ of the variation in on-time full vaccination across communities was explained by both individual and community level factors included in model-IV (Table 3).

\section{Multicollinearity and interaction effect}

Multicollinearity was checked for those variables included in the final model using VIF. Accordingly, the VIF for all predictor variables included in the final model was below 10 indicating absence of multicollinearity among the predictor variables. Similarly, interactions between community and individual level variables were tested and there was no statistically significant cross-level interaction.

\section{Comparison of models and model fit statistics}

Akakie Information Criterion (AIC) was used to compare the models. The AIC values for each subsequent models were compared and Model-IV with lowest value of AIC was considered to be the better model (Table 3).

Finally, goodness of fit test was done for the final model. The Hosmer and Lemeshow test was statistically insignificant indicating that the final model fits the data very well ( $P$-value: 0.279$)$.

\section{Discussion}

Timely vaccination is very important to get the maximum benefit of the vaccine. As the health system is mostly focusing on the completion of vaccines, timely completion of recommended vaccines is important for evaluating the effectiveness of immunization programs in Ethiopia. In this study a total of 774 children nested within 10 clusters were included in the analysis after excluding 38 unverifiable records. The analysis showed that timely vaccination coverage is low in the study area. The results of the study also indicated that individual and community level factors were significantly associated with on-time full vaccination.

The findings of this study indicated that around twothird of children were fully vaccinated which is far behind the national target [5]. This finding is also relatively lower than the findings of studies in Dessie [21], Woldia [22] and Markos [26] towns. This could be explained by the difference in information source for outcome measurement where we have used objective measures to ascertain vaccination status of children from the EPI cards and health facility registers only. In this study, one third of children had not completed their vaccination and the coverage rate for specific vaccines declined for subsequent doses of vaccines with high dropout rates. This finding corroborates with other evidences where missing measles and third doses of polio and pentavalent vaccine were the main reason for not being fully vaccinated [3, $24,43]$. In addition, the study indicated different coverage's for specific vaccines provided in the same vaccination visits. This finding is in line with other studies $[21,26]$. This might be related with unavailability of some specific vaccines or supplies especially for the newly introduced vaccines like Rota and PCV during vaccination sessions. Service availability and readiness assessment report of 2018 also showed that availability of vaccines in health facilities ranges between 28 and 30\% [59].

In terms of timeliness, we found that that almost twothird of the children were not vaccinated on-time either being early or late from the recommended time schedules. These findings corroborate the findings of other studies most of which reported higher proportion of delays in child vaccinations [10, 13, 27, 33, 49, 60-64] This study also pointed out that only half of fully vaccinated children were timely for all the vaccine doses. This indicates that merely relying on vaccination coverage overestimates population immunity, as it does not account for delays in protection and extended susceptibility to preventable diseases. Another reports in the literature also concur that timely vaccination coverage is a better performance metric for routine immunization services than crude vaccination rates alone $[53,61]$. As such, untimely vaccinations are likely to contribute greatly to the preventable disease burden in Ethiopia, allowing for transmission among those in the age group at which they are the most vulnerable to severe disease.

In the present study timely vaccination coverage was not only low, but also it declines as one goes from penta I to penta III and measles vaccine doses which is consistent with other evidences [32, 45, 46]. This might be due to increased caregiver's workload with other activities while the child gets older and thereby might not remember vaccination appointments of their children.

This study also found that a substantial number of children started their routine vaccination much earlier than the recommended age. Similar result has been reported elsewhere [43]. We found a high proportion of children vaccinated before 9 months of age for measles 
which may be related with the measles open vial policy issues in which, the measles multi-dose vial has not been opened daily unless sufficient numbers of children were found in the vaccination site; be it in a static or outreach setting. According to the Advisory Committee on Immunization Practice recommendation, these earlier vaccine doses leads to low sero-conversion rates with less child protection from vaccine-preventable disease and the vaccine dose should be repeated [42].

This study also demonstrated that there was a delay in all vaccine types showing that many Ethiopian children are receiving their vaccinations later than recommended, leaving them unnecessarily vulnerable to disease for extended periods. The implication of delay in receipt of vaccines is that a pool of children with incomplete or no immunization may build up [62]. The presence of such a pool of susceptible children predisposes to outbreaks of vaccine preventable diseases [13].

This study finally assessed the individual and community level factors associated with on-time full vaccination. At the individual level the variables age of caregiver, educational status, household wealth index, ANC and PNC service utilization were significantly associated with on-time full vaccination. The study showed that, as the caregivers get older the odds of timely full vaccination decreases. This finding is consistent with findings from Belgium [10], China [30] and Saudi Arabia [37]. This difference could be explained by the reason that the younger caregiver would possibly have a better utilization of health care which may lead to an increased probability of vaccinating their child on-time [30]. On the other hand no significant effect was reported from studies in Burkina Faso [31] Uganda [13] and Pakistan [52].

On-time full vaccination coverage was higher among children of caregivers with high educational level. Several studies also support the finding of higher educational level being related to timely adherence to the vaccination schedules $[13,16,31,32,50$, $51,63,65]$. The possible reason for this may be related with the fact that the low education level can hinder the caregiver's communication with health workers and might influence caregiver's awareness to seek and take advantage of public health services including child vaccination.

Household wealth index was also found to be a significant predictor of on-time full vaccination in this study, with a better timeliness of vaccination among children from the richest households. This finding is consistent with findings from other countries $[10,13,16,50,51$, $61,66]$. Though immunization services in Ethiopia are completely free of charge, the indirect cost of vaccination, such as income loss and transportation cost, might be associated with the low demand for vaccination especially for poorest households [50].

ANC visit of four or more at the health facility predicts better timely completion of child vaccination as it has been reported from other study settings [13, 61, 65]. It is possible that mothers who attended ANC visits at health facilities may be more frequent users of health facilities and services including vaccination for children. In this study on-time full vaccination coverage was high among children whose mothers attended postnatal care [43]. This is expected as they can get counselling about vaccination during PNC visits and their children have more chances of getting the vaccines than those who do not make any follow-up contact for PNC service.

At community level, the variables distance to the vaccination site and place of vaccination were significantly associated while place of residence didn't show significant association with on-time full vaccination. In this study, distance to the vaccination site was negatively associated with on-time full vaccination. This finding is consistent with studies in Burkina Faso [31], China [30] and Tanzania [36]. Thus, this further strengthened the argument that the time spent to reach the vaccination site expenses a high opportunity cost to caregivers by creating the need for multiple visits, especially when vaccine vials were not opened for a small number of children like BCG and measles vaccines.

The findings of this study also indicated that timely vaccination was more likely if the child was vaccinated in hospitals than health posts. This study is consistent with a study in Lebanon [60]. This may reflect that health services, mainly having a better utilization of vaccination services, were more preferable and accessible to caregivers. National reports also indicated that child vaccination as an outreach service was not commonly offered on daily basis which is the case at health post level $[59,67]$.

In our study, place of residence have no significant association with on-time full vaccination. In Ethiopia, urban areas might have easier access to health services and better transportation available whereas the introduction of the health extension program might have increased access to vaccinations in rural areas with wellestablished rural outreach immunization efforts, which may account for the insignificant results in this study. This finding corroborates with a finding from Pakistan where residence has no effect on timeliness of vaccination [52]. This finding is inconsistent with other findings from Bangladesh [50], Vietnam [51] and Nigeria [68] where children from urban areas have better timeliness for vaccination. This was justified by the reason that health facilities are more proximal to clients in the urban community than the rural community. On contrary, in a study from Burkina Faso children from rural 
have better timeliness for Penta III and measles vaccination [31]. It was explained that although the urban area has a better health infrastructure compared to the surrounding villages, caregivers need to take their children to the health facilities by their own initiative, while rural villages are visited by an outreach vaccination team each month.

In addition, we found evidence of clustering effects of timely full vaccination at community (Kebele) level, such that children from the same communities tended to have similar vaccination status. This suggests that public health programs designed to improve timeliness of vaccination should address people and the communities in which they live [69].

The results of this study should be interpreted taking in to account the following limitations. Though Gondar city administration has urban and rural kebeles, the study was restricted to children having a history of routine vaccination in one city administration. So, the study findings might not be generalizable to children in all regions across Ethiopia. Our study participants might have also introduced recall bias in remembering the frequency of maternal health service utilization factors and the reasons for untimely vaccinations. To reduce recall bias, we ascertained vaccination outcomes objectively from EPI cards and EPI registers of health facilities. Despite these limitations, this study is a community-based survey that would be more representative of the children population. Similarly, the analysis considered the clustered nature of the data by applying multi-level modeling.

\section{Implications for practice and research}

The evidence presented in this study highlighted that strengthening immunization program requires special efforts directed towards the inclusion of timeliness of vaccination as another indicator to monitor the performance of the EPI program in Ethiopia. Future strategies and studies should also develop and test intervention programs to improve timely vaccination for children in developing countries like Ethiopia. In addition, conducting a nationally representative survey about timeliness of vaccination is important to monitor the quality of the EPI program.

\section{Conclusion}

In this study, full vaccination coverage was relatively low and untimely vaccination was found to be high. At individual level the variables caregiver's age, caregiver's education, household wealth index, ANC and PNC service utilization were the significant factors affecting on-time full vaccination. In addition, at community level the variables distance to vaccination site and place of vaccination were the significant predictors of on-time full vaccination. Therefore, health facility vaccinators should focus on timeliness of vaccination to minimize early and delayed administration of vaccines. Moreover, targeted interventions should be implemented among the older, uneducated and caregivers with low socio-economic status. In addition, strengthening the quality of ANC and PNC services is also important. At health post level, emphasis should be given for daily vaccination service provision.

\section{Abbreviations \\ AIC: Akakie Information Criterion; ANC: Ante Natal Care; AOR: Adjusted Odds Ratio; BCG: Bacille Calmette Guérin; Cl: Confidence Interval; DTP: Ddiphtheria- Tetanus-Pertussis; EDHS: Ethiopian Demographic and Health Survey; EPHI: Ethiopian Public Health Institue: EPI: Expanded Program on Immunization; FMOH: Federal Ministry of Health; ICC: Intra Class Correlation Coefficient; IPV: Inactivated Polio Vaccine; OPV: Oral Polio Vaccine; PCA: Principal Component Analysis; PCV: Proportional Change in Variance; PNC: Post Natal care; VIF: Variance Inflation Factor; VPDs: Vaccine Preventable Diseases; WHO: World Health Organization}

\section{Authors' contributions}

ZAM conceived the study. KAG, MW and BT guided the design and conduct of the study. All the authors were involved in data analysis and manuscript write-up. All authors read and approved the final manuscript.

\section{Funding}

This study was supported by University of Gondar (DDCF project). University of Gondar (DDCF project) has no any role in the design of the study, collection, analysis, interpretation of data and in writing the manuscript.

\section{Availability of data and materials}

The datasets used for this particular study will be available from the corresponding author up on reasonable request.

\section{Ethics approval and consent to participate}

This study obtained ethical approval from University of Gondar Institutional Ethical Review Board (ref no: O/N/P/RCS/05/060/2018). In addition, study permission was acquired at all levels and informed written consent was obtained from study participants.

Consent for publication

Not applicable.

\section{Competing interests}

The authors declare that they have no competing interests.

\section{Author details}

${ }^{1}$ Department of Health Informatics, Institute of Public Health, University of Gondar, Gondar, Ethiopia. ${ }^{2}$ Health System Directorate, Federal Ministry of Health, Addis Ababa, Ethiopia. ${ }^{3}$ Department of Epidemiology and

Biostatistics, Institute of Public Health, University of Gondar, Gondar, Ethiopia. ${ }^{4}$ Department of Biomedical Informatics, Vanderbilt University Medical Center, Nashville, USA.

Received: 13 January 2020 Accepted: 17 May 2020

Published online: 11 June 2020

\section{References}

1. WHO, UNICEF and World Bank. State of the world's vaccines and immunization. 3rd ed. Geneva: World Health Organization; 2009.

2. Shen K, Fields R, McQuestion M. The future of routine immunization in the developing world: Challenges and opportunities. Glob Health Sci Pract. 2014;2(4):381-94. https://doi.org/10.9745/GHSP-D-14-00137.

3. GAVI. Analysis of Coverage of Fully Immunized Child (FIC), Associated Factors, Outcomes, and Impact Using Routinely Collected Population Cohort Data 2001-2014, 2015.

4. FMOH. National Strategy for Newborn and Child Survival in Ethiopia: 2015/ 16-2019/20.

5. $\mathrm{FMOH}$. Ethiopia national expanded programme on immunization Comprehensive multi-year plan 2016-2020. 
6. FMOH. Health Sector Transformation Plan, 2015.

7. World Health Organization. Immunization, Vaccines and Biologicals, IVB Catalogue, 2017. Available from: https://extranet.who.int/ivb_docs/reports/ catalogue. Accessed 03 Sep 2019.

8. Dolan B, Carnahan E, Shearer C, Beylerian N, Thompson J, Gilbert S, et al. Redefining vaccination coverage and timeliness measures using electronic immunization registry data in low- and middle-income countries. Vaccine. 2019;37(13):1859-67.

9. Hu Y, Chen Y. Timeliness of childhood primary immunization and risk factors related with delays: evidence from the 2014 Zhejiang Provincial vaccination coverage survey. Int J Environ Res Public Health. 2017;14(9):113. https://doi.org/10.3390/ijerph14091086.

10. Lernout T, Theeten $\mathrm{H}$, Hens $\mathrm{N}$, Braeckman T, Roelants $\mathrm{M}$, Hoppenbrouwers $K$, et al. Timeliness of infant vaccination and factors related with delay in Flanders, Belgium. Vaccine. 2013/11/21. 2014;32(2):284-9.

11. Kiely M, Boulianne N, Talbot D, Ouakki M, Guay M, Landry M, et al. Impact of vaccine delays at the 2, 4,6 and 12 month visits on incomplete vaccination status by 24 months of age in Quebec. Canada: BMC Public Health; 2018.

12. Mbengue MAS, Mboup A, Ly ID, Faye A, Camara FBN, Thiam M, et al. Vaccination coverage and immunization timeliness among children aged 12-23 months in Senegal: a Kaplan-Meier and Cox regression analysis approach. Pan Afr Med J. 2017;27(Suppl 3):8.

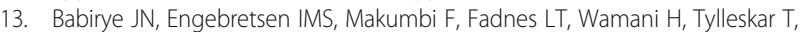
et al. Timeliness of childhood vaccinations in Kampala Uganda: a community-based cross-sectional study. PLoS One. 2012;7(4):e35432.

14. Machingaidze S, Wiysonge CS, Hussey GD. Strengthening the expanded programme on immunization in Africa: looking beyond 2015. PLoS Med. 2013;10(3):e1001405.

15. World Health Organization. Immunization coverage. Fact Sheets. 2018; Available from: https://www.who.int/en/news-room/fact-sheets/detail/ immunization-coverage. Accessed 11 Oct 2019.

16. Akmatov MK, Mikolajczyk RT. Timeliness of childhood vaccinations in 31 low and middle-income countries. J Epidemiol Community Heal. 2011/05/10. 2012;66(7):e14

17. Zida-Compaore WIC, Ekouevi DK, Gbeasor-Komlanvi FA, Sewu EK, Blatome T, Gbadoe AD, et al. Immunization coverage and factors associated with incomplete vaccination in children aged 12 to 59 months in health structures in Lomé. BMC Res Notes. 2019;12(1):84.

18. CSA and ICF International. Ethiopian Demographic and Health Survey, 2016.

19. EPHI and FMOH. Mini Demographic and Health Survey, 2019.

20. Workina A, Seid S, Moga TT. Reason for incomplete vaccination and associated factors among children aged 12-23 months in Serbo Town, Jimma Zone, Oromia Region, Southwest Ethiopia. Adv Res J Multidis Discov. 2018:32(14):79-84.

21. Lake MW, Boulanger L, Wasswa P, Merbratu M, Fenta A. Factors for low routine immunization performance; a community based cross sectional study in Dessie town, south Wollo zone, Ethiopia, 2014. Advances Appl Sci. 2016;1 (1):7-17.

22. Birara A. Coverage, Opportunity and Challenges of expanded Program on Immunization among 12-23 Months old Children in Woldia Town, Northeast, Ethiopia; 2018. p. 1-14.

23. Animaw W, Taye W, Merdekios B, Tilahun M, Ayele G. Expanded program of immunization coverage and associated factors among children age 12-23 months in Arba Minch town and Zuria District, Southern Ethiopia, 2013. BMC Public Health. 2014;14(1):464.

24. Yismaw AE, Assimamaw NT, Bayu NH, Mekonen SS. Incomplete childhood vaccination and associated factors among children aged 12-23 months in Gondar city administration, northwest, Ethiopia 2018. BMC Res Notes. 2019; 12(1):241.

25. Nina B, Tefera Y, Wagner L, Boulton L. Vaccine hesitancy among caregivers and association with childhood vaccination timeliness in Addis Ababa Ethiopia. Hum Vaccin Immunother. 2018;14(10):2340-7. https://doi.org/10. 1080/21645515.2018.1480242.

26. Gualu T, Dilie A. Vaccination coverage and associated factors among children aged 12-23 months in Debre Markos Town, Amhara Regional State, Ethiopia. Adv Public Health. 2017. https://doi.org/10.1155/2017/ 5352847. p. 1-4.

27. Tauil M d C, Ferreira VL d R, APS S, Costa ÂA, Inenami M, Waldman EA Vaccination coverage according to doses received and timely administered based on an electronic immunization registry, Araraquara-SP, Brazil, 2012-2014. Epidemiol e Serv saude Rev do Sist Unico Saude do Bras. 2017;26:835-46.
28. Fadnes LT, Nankabirwa V, Sommerfelt $H$, Tylleskär T, Tumwine JK, Engebretsen IMS. Is vaccination coverage a good indicator of ageappropriate vaccination? A prospective study from Uganda. Vaccine. 2011; 29(19):3564-70

29. Saini V, MacDonald SE, McNeil DA, McDonald SW, Kellner JD, Edwards SA, et al. Timeliness and completeness of routine childhood vaccinations in children by two years of age in Alberta, Canada. Can J Public Heal. 2017; 108(2):e124-8.

30. Hu Y, Chen Y, Guo J, Tang X, Shen L. Completeness and timeliness of vaccination and determinants for low and late uptake among young children in eastern China. Hum Vaccin Immunother. 2014;10(5):1408-15.

31. Schoeps A, Ouedraogo N, Kagone M, Sie A, Muller O, Becher H. Sociodemographic determinants of timely adherence to $B C G$, Penta3, measles, and complete vaccination schedule in Burkina Faso. Vaccine. 2013/11/05. 2013;32(1):96-102.

32. Odutola A, Afolabi MO, Ogundare EO, Lowe-Jallow YN, Worwui A, Okebe J, et al. Risk factors for delay in age-appropriate vaccinations among Gambian children. BMC Health Serv Res. 2015;15(1):346.

33. Scott S, Odutola A, Mackenzie G, Fulford T, Afolabi MO, Jallow YL, et al. Coverage and timing of children's vaccination: An evaluation of the expanded programme on immunisation in the Gambia. PLoS One. 2014; 9(9):e107280.

34. Mutua MK, Kimani-Murage E, Ngomi N, Ravn H, Mwaniki P, Echoka E. Fully immunized child: coverage, timing and sequencing of routine immunization in an urban poor settlement in Nairobi. Trop Med Health: Kenya; 2016.

35. Nalley J, Maduka O. Completeness and timeliness of immunization among children aged 12 to 23 months in south-South Nigeria. J Community Med Prim Heal Care. 2019:31(1):22-31.

36. Le Polain de Waroux O, Schellenberg JRA, Manzi F, Mrisho M, Shirima K Mshinda $\mathrm{H}$, et al. Timeliness and completeness of vaccination and risk factors for low and late vaccine uptake in young children living in rural southern Tanzania. Int Health. 2013:5(2):139-47.

37. Alrowaili GZR, Dar UF, Bandy AH. May we improve vaccine timeliness among children ? A cross sectional survey in northern Saudi Arabia. J Family Community Med. 2019;26(2):113-7. https://doi.org/10.4103/ffcm.JFCM_153_18.

38. Gizachew M, Birhane N, Mekonnen M, Jember A. A Community Based Assessment of the Immunization Coverage and the Associated Factors of Children Aged 12-23 Months at Gondar Town, Northwest Ethiopia. EC Microbiol; 2015. p. 247-60.

39. Gondar City Health Department, Gondar city administration health office report, 2018.

40. $\mathrm{FMOH}$. National Expanded Program on immunization implementation guideline. Revised Edition,2015.

41. Negussie A, Kassahun W, Assegid S, Hagan AK, et al. Factors associated with incomplete childhood immunization in Arbegona district, southern Ethiopia: A case - Control study. BMC Public Health. 2016;16(27). https://doi.org/10. 1186/s12889-015-2678-1.

42. CDC. General Recommendations on Immunization Recommendations of the Advisory Committee on Immunization Practices (ACIP), 2011.

43. Adetifa IMO, Karia B, Mutuku A, Bwanaali T, Makumi A, Wafula J, et al. Coverage and timeliness of vaccination and the validity of routine estimates: insights from a vaccine registry in Kenya. Vaccine. 2018;36(52): 7965-74.

44. Zaidi SMA, Khowaja S, Dharma VK, Khan AJ, Chandir S. Coverage, timeliness, and determinants of immunization completion in Pakistan: evidence from the demographic and health survey (2006-07). Hum Vaccines Immunother. 2014;10(6):1712-20.

45. Marefiaw TA, Yenesew MA, Mihirete KM. Age-appropriate vaccination coverage and its associated factors for pentavalent 1-3 and measles vaccine doses, in Northeast Ethiopia: a community-based cross-sectional study. PLoS One. 2019;14(8):1-19.

46. Laryea DO, Abbeyquaye Parbie E, Frimpong E. Timeliness of childhood vaccine uptake among children attending a tertiary health service facilitybased immunisation clinic in Ghana. BMC Public Health. 2014;14(1):90.

47. WHO. Recommended Routine Immunizations for Children, 2017. Available from: https://www.who.int/immunization/policy/immunization_tables/en/. Accessed 20 Oct 2019

48. Veerasingam P, Grant CC, Chelimo C, Philipson K, Gilchrist CA, Berry S, et al. Vaccine education during pregnancy and timeliness of infant immunization. Pediatrics. 2017;140(3):e20163727. 
49. Stein-Zamir C, Israeli A. Timeliness and completeness of routine childhood vaccinations in young children residing in a district with recurrent vaccinepreventable disease outbreaks, Jerusalem, Israel. Eurosurveillance. 2019;24(6):1-8.

50. Sheikh N, Sultana M, Ali N, Akram R, Mahumud R, Asaduzzaman M, et al. Coverage, Timelines, and Determinants of Incomplete Immunization in Bangladesh. Trop Med Infect Dis. 2018;3(3):72.

51. Minh An DT, Lee JK, Van Minh HV, Trang NTH, Huong NTT, Nam YS, et al. Timely immunization completion among children in Vietnam from 2000 to 2011: a multilevel analysis of individual and contextual factors. Glob Health Action. 2016;9(1):29189.

52. Noh JW, Kim YM, Akram N, Yoo KB, Cheon J, Lee LJ, et al. Determinants of timeliness in early childhood vaccination among mothers with vaccination cards in Sindh province, Pakistan: a secondary analysis of cross-sectional survey data. BMJ Open. 2019;9(9):1-9.

53. Chiabi A, Nguefack FD, Njapndounke F, Kobela M, Kenfack K, Nguefack S, et al. Vaccination of infants aged 0 to 11 months at the Yaounde Gynaecoobstetric and pediatric hospital in Cameroon: how complete and how timely? BMC Pediatr. 2017;17(1):1-7.

54. Shea R, Johnson $\mathrm{K}$. The DHS wealth index. DHS comparative reports no. 6 . ORC Macro: Calverton, Maryland; 2004.

55. Hosmer D, Lemeshow S. Applied Logistic Regression. Second Edition; 2000.

56. Stawski RS. Multilevel analysis: An introduction to basic and advanced multilevel modeling (2nd edition). Struct Equ Model A Multidiscip J. 2013; 20(3):541-50.

57. Akinwande MO, Dikko HG, Samson A. Variance inflation factor: as a condition for the inclusion of suppressor variable(s) in regression analysis. Open J Stat. 2015;05(07):754-67.

58. Hamaker EL, van Hattum P, Kuiper RM, Hoijtink H. Model selection based on information criteria in multilevel modeling. In Hox JJ, Roberts JK, editors. European Association for Methodology series. Handbook for advanced multilevel analysis; 2011. p. 231-55.

59. EPHI. Services Availability and Readiness Assessment ( SARA ) 2018 Final Report, 2018.

60. Mansour Z, Said R, Brandt L, Khachan J, Rady A, Fahmy K, et al. Factors affecting age-appropriate timeliness of vaccination coverage among children in Lebanon. Gates Open Res. 2018;2(May):1-13.

61. Vasudevan L, Labrique AB, Mehra S, Wu L, Levine O, Feikin D, et al. Materna determinants of timely vaccination coverage among infants in rural Bangladesh. Vaccine. 2014;32(42):5514-9.

62. Clark A, Sanderson C. Timing of children's vaccinations in 45 low-income and middle-income countries: an analysis of survey data. Lancet. 2009/03/ 24. 2009;373(9674):1543-9.

63. Mohammadbeigi A, Mokhtari M, Zahraei SM, Eshrati B, Rejali M. Survival analysis for predictive factors of delay vaccination in iranian children. Int J Prev Med. 2015;6:119.

64. Fadnes LT, Jackson D, Engebretsen IMS, Zembe W, Sanders D, Sommerfelt $\mathrm{H}$, et al. Vaccination coverage and timeliness in three south African areas: a prospective study. BMC Public Health. 2011;11:404.

65. Noh JW, Kim YM, Akram N, Yoo KB, Park J, Cheon J, et al. Factors affecting complete and timely childhood immunization coverage in Sindh, Pakistan A secondary analysis of crosssectional survey data. PLoS One. 2018;13(10): e0206766.

66. Boulton ML, Carlson BF, Wagner AL, Porth JM, Gebremeskel B, Abeje Y. Vaccination timeliness among newborns and infants in Ethiopia. PLoS One. 2019;14(2):e0212408.

67. Ethiopian Public Health Institute. Ethiopian national immunization coverage survey; 2012.

68. Sibeudu FT, Uzochukwu BS, Onwujekwe OE. Rural-urban comparison of routine immunization utilization and its determinants in communities in Anambra states, Nigeria. SAGE Open Med. 2019;7:205031211882389.

69. Wiysonge CS, Uthman OA, Ndumbe PM, Hussey GD. Individual and Contextual Factors Associated with Low Childhood Immunisation Coverage in Sub-Saharan Africa: A Multilevel Analysis. PLoS One. 2012;7(5). https://doi. org/10.1371/journal.pone.0037905.

\section{Publisher's Note}

Springer Nature remains neutral with regard to jurisdictional claims in published maps and institutional affiliations.

\section{Ready to submit your research? Choose BMC and benefit from}

- fast, convenient online submission

- thorough peer review by experienced researchers in your field

- rapid publication on acceptance

- support for research data, including large and complex data types

- gold Open Access which fosters wider collaboration and increased citations

- maximum visibility for your research: over $100 \mathrm{M}$ website views per year

At $\mathrm{BMC}$, research is always in progress.

Learn more biomedcentral.com/submissions 